\title{
Analyse de classification hiérarchique et qualité de vie
}

\author{
Mélanie Vachon Dominic Beaulieu-Prévost Amélie Ouellette Marie Achille \\ Université de Montréal
}

\begin{abstract}
This article is a theoretical as well as a practical overview of the hierarchical cluster analysis method. First, the method is presented according to the seven-step procedure proposed by Rapkin and Luke (1993). The stakes associated with each of these steps are discussed and the most currently used methods are detailed. Then, a study about the quality of life of type I diabetic patients who have received a transplant serves as an example. In this study, the hierarchical cluster procedure yielded two profiles of quality of life among these patients. Moreover, the class validation suggests some determinants of quality of life, including the type of transplant that was received. Finally, hierarchical cluster analysis is discussed in the present context of research in the field of psychology.
\end{abstract}

Cet article est un survol, à la fois théorique et pratique, de l'analyse de classification hiérarchique. La démarche présentée correspond à la procédure en sept étapes proposée par Rapkin et Luke (1993). Les enjeux associés à chacune de ces étapes sont discutés et les méthodes couramment utilisées y sont explicitées. L'analyse de classification hiérarchique est également exemplifiée à l'aide une étude sur la qualité de vie des patients diabétiques de type I ayant reçu une greffe. Dans le cadre de cette étude, l'analyse de classification hiérarchique a permis de dégager deux profils de qualité de vie chez les patients greffés. Également, la validation des classes obtenues suggère certains déterminants de la qualité de vie chez cette population, notamment le type de greffe reçu. Finalement, l'analyse de classification hiérarchique est située dans le contexte actuel de la recherche en psychologie.

L'analyse de classification, connue sous le nom de "cluster analysis", est un terme générique utilisé pour désigner une variété de techniques d'analyses statistiques (Scheibler \& Schneider, 1985). Ces méthodes sont généralement utilisées pour grouper des objets, des personnes, des concepts ou des stimuli dans des groupes homogènes sur la base de leur similarité. Ces techniques de classification sont particulièrement utiles dans le cadre d'investigations exploratoires afin de dégager des tendances générales au sein des données et de suggérer des pistes d'analyses futures (Kos \& Psenicka, 2000). L'objectif global des analyses de classification est d'identifier des classes à l'intérieur d'un ensemble d'entités mélangées que l'on croit appartenir à des populations différentes. Précisément, elles visent à (1) construire un schème contextuel pour la classification d'entités, (2) générer des hypothèses, (3) tester des classes hypothétiques à l'intérieur d'un échantillon et à (4) identifier des sous-groupes homogènes caractérisés par des tendances utiles pour des prédictions futures (Lorr, 1983). Concrètement, ce type d'analyse a permis d'évaluer la classification utilisée dans le manuel diagnostique et statistique des troubles mentaux (DSM-III), ce qui a offert une première base empirique et statistique à la classification de certaines maladies mentales (Skinner, 1981).

\section{L'analyse de classification hiérarchique}

Les analyses de classification se divisent en cinq familles, soit (1) l'agglomération hiérarchique, (2) la division hiérarchique, (3) la partition interactive, (4) la recherche de densité et (5) les variantes de l'analyse factorielle. La méthode de l'agglomération, désignée de façon interchangeable par le terme «analyse de classification hiérarchique», est celle qui retient ici notre attention puisqu'elle est la plus utilisée. Environ les deux tiers de toutes les utilisations appliquées des analyses de classification ont impliqué l'analyse de classification hiérarchique (Blashfield \& Aldenderfer, 1988). Cette analyse permet de produire autant de classes qu'il y a d'entités (e.g. participants) dans la base de données (Milligan, 1981). Au départ, chaque entité représente une 
sous-classe. Ces sous-classes sont ensuite regroupées sur la base de leur similarité, jusqu'à ce chaque entité fasse partie d'un groupe (Blashfield \& Aldenderfer, 1988).

\section{Variables internes et externes}

Les groupes sont formés en fonction de la similarité des entités au niveau de variables internes prédéterminées. Grossièrement, il s'agit de choisir à partir de quelles informations on désire que les classes soient formées (Beaulieu-Prévost, 2002). Ces variables internes peuvent être considérées comme les composantes d'un profil ou d'une typologie. Le choix des variables internes est motivé par ce qui importe au chercheur de savoir.

D'autre part, les variables externes peuvent se définir comme étant les variables sur lesquelles se distinguent les profils. Une fois les typologies constituées, un chercheur peut s'intéresser aux variables externes possiblement en lien avec chacune des typologies. Bref, les classes d'observations étant dégagées (à l'aide des variables internes), il est possible d'investiguer ces classes (à l'aide des variables externes) afin d'identifier certains déterminants des regroupements (Kos \& Psenicka, 2000).

\section{La procédure}

Toute procédure de classification nécessite plusieurs étapes. À cet effet, Rapkin \& Luke (1993) ont suggéré une procédure en sept étapes successives, soit l'opérationnalisation des variables, les analyses descriptives, les décisions pré-classification, le choix du nombre optimal de classes, l'évaluation de la stabilité de la classification, l'interprétation des classes et la validation des classes (Beaulieu-Prévost, 2002). Chacune de ces étapes est décrite dans les paragraphes qui suivent.

\section{Opérationnalisation des variables}

Comme dans la plupart des analyses statistiques, la classification hiérarchique nécessite que les variables soient opérationnalisées. Cette étape consiste donc à préciser en termes mesurables chacune des variables internes et externes qui seront incluses dans l'analyse.

\section{Analyses descriptives}

Avant de débuter l'analyse de classification hiérarchique, il importe d'effectuer les analyses descriptives appropriées dans le cadre de l'étude en cours (Rapkin et Luke, 1993). Ces analyses permettent, entre autres, de s'assurer de la normalité des distributions et d'identifier les données possiblement aberrantes.

\section{Les décisions pré-classification}

Une analyse de classification hiérarchique implique trois décisions importantes, à savoir: la standardisation ou non des données, la méthode d'agrégation, ainsi que la façon de calculer l'espace métrique.

\section{Standardisation}

La première décision pré-classification à prendre est celle de standardiser ou non les données. Plusieurs auteurs ont discuté des avantages et désavantages de la standardisation (Beaulieu-Prévost, 2002). L'avantage principal de la standardisation est qu'elle permet d'inclure, dans une même classification, des variables internes mesurées à l'aide d'échelles non comparables. En fait, peu importe la façon dont elle est mesurée, chaque variable aura un poids équivalent dans la classification (Beaulieu-Prévost, 2002). Les données de l'exemple qui suivra seront donc standardisées par des valeurs-étalon (Z).

\section{Méthode d'agrégation}

La méthode d'agrégation se définit comme étant la méthode utilisée pour regrouper les entités (participants, stimuli) les unes avec les autres. Il existe plusieurs méthodes d'agrégation, les principales étant la méthode $\mathrm{du}$ lien simple, du lien complet, du lien moyen, ainsi que la méthode de Ward. Chacune de ces méthodes présentent des avantages et inconvénients. Entre autres, certains auteurs ont mentionné que la méthode du lien simple est trop libérale puisqu'elle regroupe deux classes même si une seule entité de chacune de ces classes sont similaires. D'autre part, la méthode du lien complet semble trop conservatrice; elle requiert une similarité complète entre toutes les entités de chacune des classes afin de les regrouper (Blashfield \& Aldenderfer, 1988).

La méthode de Ward semble quant à elle avoir démontré des résultats très puissants comparativement aux solutions obtenues par les autres méthodes (Morey, Blashfield, \& Skinner, 1983). Elle est également largement utilisée dans la plupart des études en sciences humaines. Cette méthode définit une classe comme étant un groupe d'entités dans lequel la variance entre les membres est relativement petite (Blashfield \& Aldenderfer, 1988). Les classes sont progressivement formées en fonction du principe de la minimisation de la variance (Morey, Blashfield, \& Skinner, 1983). La méthode de Ward semble créer des classes relativement égales (Blashfield \& Aldenderfer, 1988) et est particulièrement utile lorsqu'on s'attend à des classes bien distinctes (Lorr, 1983). C'est donc méthode de Ward qui sera utilisée l'exemple qui suivra.

\section{L'espace métrique}

L'espace métrique permet d'évaluer le degré auquel les entités sont considérées comme étant similaires (Morey, Blashfield, \& Skinner, 1983). Il existe quatre types de mesure de l'espace métrique, mais seulement deux sont utilisées en sciences humaines. Il s'agit des coefficients de corrélation et des coefficients de distance (Blashfield \& Aldenderfer, 1988) D'abord, les coefficients de corrélation sont surtout utilisés pour classifier des variables et leur 
utilisation pour la classification d'individus s'avère problématique (Aldenderfer \& Blashfield, 1984; dans Beaulieu-Prévost, 2002). Dans le contexte de la psychologie, il est donc plus pertinent de s'attarder aux coefficients de distance. Il existe plusieurs mesures pour évaluer la distance métrique (Blashfield \& Aldenderfer, 1988), mais les plus couramment utilisées semblent être la distance euclidienne, la distance de Manhattan et la distance de Mahalanobis (Rapkin \& Luke, 1993; dans Beaulieu-Prévost, 2002).

La distance euclidienne demeure la mesure de similarité la plus populaire (Morey, Blashfield, \& Skinner, 1983). Elle représente la distance géographique la plus courte entre deux points dans un espace multidimensionnel (Beaulieu-Prévost, 2002). Cet espace contient autant de dimensions qu'il y a de variables internes. Ainsi, si une classification est effectuée sur la base de trois variables internes, chaque participant représentera un point dans un espace tridimensionnel. La distance euclidienne permet de mesurer la distance entre ces points pour, par la suite, former des groupes avec les points les plus rapprochés. Puisqu'elle démontre une bonne performance lorsque combinée à la méthode d'agrégation de Ward (Beaulieu-Prévost, 2002), c'est la distance euclidienne qui sera utilisée comme mesure de similarité dans l'étude citée en exemple.

\section{Le choix du nombre optimal de classes}

La sélection du nombre de classes que comprendra la solution finale est un aspect crucial de l'évaluation des solutions proposées par l'analyse de classification hiérarchique (Barbaranelli, 2001). Les chercheurs sont confrontés au problème d'obtenir soit trop ou trop peu de classes. Toutefois, bien qu'il n'existe pas $\mathrm{d}$ 'indice unique pour déterminer le nombre optimal de classes, de nombreux critères statistiques peuvent être utilisés pour faciliter cette décision. Premièrement, il est possible de prendre une décision en fonction du seuil de signification des ANOVAs effectuées sur les variables internes (Beaulieu-Prévost, 2002). Si les profils obtenus se distinguent largement $(p<.001)$ sur les variables utilisées pour la classification, la solution proposée est probablement pertinente. Deuxièmement, la facilité $\mathrm{d}$ 'interprétation est également un critère qui nous renseigne sur le nombre de classe adéquat. Il importe donc de se questionner sur la pertinence théorique des profils obtenus. Finalement, la taille de l'échantillon doit aussi être prise en considération. Plus l'échantillon est grand, plus il fait du sens d'opter pour plusieurs classes. Dans le cadre de la présente étude, le niveau de signification des ANOVAs, la taille de l'échantillon $(\mathrm{N}=43)$ ainsi que l'interprétabilité des classes ont fait en sorte qu'il était plus approprié d'opter pour une solution à deux classes.

\section{Évaluation de la stabilité des classes}

La reproductibilité des phénomènes est fondamentale à l'objectivité scientifique (Breckenridge, 1989). La classification hiérarchique ne fait pas exception à la règle. Il y a de multiples façons d'évaluer la stabilité des classes. La méthode idéale serait sans doute de tenter de répliquer la même classification avec un autre échantillon issu de la même population. Cependant, cette méthode est parfois peu accessible. La façon la plus courante d'évaluer cette stabilité des classes est de refaire une analyse de classification (hiérarchique ou non) à l'aide de d'autres méthodes (e.g. une autre méthode d'agrégation ou de mesure de la distance métrique) (Lorr, 1983) à partir du même échantillon. Ainsi, on suppose que si une classification réelle existe à l'intérieur de l'échantillon, elle se manifestera peu importe la méthode utilisée (BeaulieuPrévost, 2002).

\section{Interprétations des classes}

L'interprétation des classes consiste en une description qualitative de chacun des profils créés à partir des variables internes. Il importe donc de vérifier la compréhensibilité de chacun des profils et de leur attribuer un nom (Beaulieu-Prévost, 2002). Cette étape d'interprétation sera présentée dans l'exemple subséquemment à la construction des profils.

\section{Validation des classes}

La toute dernière étape se veut un jugement sur la pertinence de la classification obtenue. En d'autres mots, il s'agit d'établir la validité des classes à l'aide des variables externes. Plusieurs méthodes permettent d'évaluer cette validité, notamment l'utilisation de critères de prédictions, de critères descriptifs ou de critères cliniques (Skinner 1981). Dans le cadre de cette étude, des critères descriptifs seront utilisés. Ces variables externes utilisées pour la validation des classes doivent être choisies en fonction de leur pertinence théorique et ce, avant d'effectuer l'analyse de classification. Ainsi, chacun des profils se doit d'être comparé sur ces variables. Dans la présente étude, cette comparaison se fera à l'aide d'ANOVAs et le seuil de signification pré-établi est de $(p<.05)$.

\section{Présente étude}

\section{Contexte}

Le diabète de type I se caractérise par une incapacité du pancréas à produire la quantité $\mathrm{d}^{\prime}$ insuline suffisante à l'organisme. Ce manque $d$ 'insuline résulte en plusieurs complications secondaires, telles que la perte de fonctionnement des reins. Actuellement, la double greffe rein-pancréas est le seul procédé qui puisse à la fois remédier à la défaillance rénale et rétablir la sécrétion endogène de l'insuline, ce qui élimine complètement le diabète (Sureshkumar et al., 2002). En effet, plusieurs recherches suggèrent que la qualité de vie $(\mathrm{QV})$ globale et le fonctionnement physique des patients ayant reçu une double greffe rein-pancréas sont supérieurs à ceux des 
patients ayant bénéficié d'une greffe rénale seule et contrôlant toujours leur diabète avec l'insuline. Toutefois, l'impact positif de la double greffe sur les aspects psychologique et social de la QV des patients n'a pas été démontré clairement (Gross et al., 2000). Cependant, Knight \& Daly, (2004) ont mentionné que les double greffés ont beaucoup plus tendance à retourner au travail après leur chirurgie que les greffés rénaux (73\% vs $23 \%$, $p<.04)$.

La littérature actuelle témoigne de l'importance de mieux comprendre l'influence des traitements recommandés pour le diabète de type I sur la QV psychologique et sociale des patients. Au-delà de la comparaison intergroupe, plusieurs questions quant à l'impact des greffes rénale et double sur qualité de vie psychosociale des patients souffrant de diabète de type I demeurent sans réponse. Entre autres :

- Existe-t-il des profils distincts de QV psychosociale chez les patients diabétiques de type I ayant reçu une greffe?

- Quelles sont les caractéristiques associées à chacun de ces profils?

- Le type de greffe est-il un déterminant de cette QV?

- Le retour au travail après la chirurgie est-il associé à une meilleure QV psychosociale?

Le but de cette étude est d'identifier des profils de QV psychosociale suite à la greffe et d'investiguer les caractéristiques déterminantes de ces profils, notamment le type de greffe reçu.

\section{Méthode}

\section{Participants et procédure}

Cinquante participants ayant reçu une double greffe rein-pancréas $(n=28)$ ou une greffe rénale $(n=22)$ ont participé à cette étude. Ils ont tous été recrutés à partir de la vaste cohorte de la clinique de transplantation de l'Hôpital Notre-Dame du CHUM. Les données ont été collectées par envoi postal.

\section{Opérationnalisation des variables}

La QV psychosociale a été mesurée à l'aide de l'échelle d'adaptation psychosociale à la maladie (version française du Psychosocial Adjustment to Illness Scale - Self Report, Derogatis, 1986). Les sept dimensions de cette échelle ont été utilisées comme variables internes afin de constituer les profils de qualité de vie psychosociale. Ces dimensions sont (1) l'attitude face aux soins de santé, (2) l'adaptation à l'environnement domestique, (3) l'adaptation à l'environnement occupationnel, (4) l'adaptation à l'environnement social, (5) la satisfaction sexuelle, (6) les relations avec la famille élargie et (7) la détresse psychologique. Chacune de ces variables est donc opérationnalisée par le score obtenu par le participant sur chacune des dimensions.

Les variables externes mises en lien avec chacun des profils sont (1) le nombre de réhospitalisations suite à la greffe, (2) le nombre de complications rapportées, (3) le type de greffe reçu, (4) le statut d'emploi et (5) la satisfaction du traitement du diabète.

\section{Démonstration de la procédure}

Une fois l'opérationnalisation des variables complétée, il faut effectuer les décisions pré-classification, soit la standardisation des variables, le choix de la méthode d'agrégation et la mesure de l'espace métrique. Dans le cadre de cette étude, la méthode d'agrégation de Ward est utilisée. La distance euclidienne permet de mesurer l'espace métrique et les données sont standardisées à l'aide de valeurs-étalon (Z).

La première étape de l'analyse consiste évidemment à démarrer le programme SPSS et à ouvrir la base de données contenant les données à analyser. Ensuite, il suffit d'ouvrir une nouvelle fenêtre de syntaxe pour y inscrire la syntaxe qui correspond à l'analyse de classification hiérarchique (voir syntaxe étape 1, tab. 1).

En effectuant cette syntaxe, un « output» complexe apparaîtra. Toutefois, cet output donne peu d'informations utiles et compréhensibles. De plus, une nouvelles variable sera automatiquement créée dans la base de données, du nom de "CLU». SPSS attribuera donc un chiffre à chacun des participants de la base de données. Ce chiffre représente le profil auquel ce participant appartient. Ensuite, il faut poursuivre l'analyse et effectuer la validation des classes à l'aide d'analyses de variance (voir syntaxe étape 2, tab.1).

\section{Interprétation des classes}

Les résultats obtenus (tab. 2) des analyses de variance effectuées sur les deux classes sont très facilement interprétables. On remarque que pour la totalité des dimensions mesurées, les patients appartenant au profil 1 obtiennent un score plus élevé que les patients appartenant au profil 2. La différence n'est cependant pas significative pour ce qui est des relations avec la famille élargie. Néanmoins, cette solution est conservée et permet de supposer que dans cet échantillon, il existe un profil de patients ayant une faible QV psychosociale (Profil 1, $n=12$ ) et un autre profil présentant un niveau de QV psychosociale plus élevée (Profil 2, $n=31$ ). Finalement, cette solution devra être répliquée par d'autres méthodes de classification afin d'être confirmée.

\section{Validation des classes à l'aide des variables externes}

Premièrement, la répartition des patients dans chacun des profils en fonction du type de greffe reçu indique que les patients ayant reçu une double greffe font partie en proportion significativement plus grande du profil de QV psychosociale élevée que du profil de QV psychosociale faible (tab. 3).

Les résultats semblent également suggérer que le statut médical n'est pas relié au niveau de QV psychosociale. À cet effet, on ne dénote pas de différence 
significative entre les deux profils (faible, élevé) pour ce qui est du nombre de complications rapportées par le participant. Par ailleurs, il semble que les patients du profil 2 (QV psychosociale élevée) aient été réhospitalisés plus souvent que les greffés rénaux pour complications relatives à la greffe. Cependant, le seuil de signification de $p<.05$ n'a pas été atteint. D'autre part, la comparaison des profils démontre une différence significative au niveau de la satisfaction du traitement et du retour au travail. Plus spécifiquement, les patients du profil 2 seraient plus satisfaits de leur traitement et travailleraient dans une plus grande proportion. Ces analyses ont donc permis de valider les classes obtenues et $d$ 'investiguer leurs associations avec les variables externes d'intérêt.

\section{Conclusion}

L'analyse de classification hiérarchique effectuée dans cette étude a permis de répondre aux questions de recherches visées. Premièrement, l'analyse a identifié deux profils de QV psychosociale à l'intérieur de l'échantillon de patients diabétiques de type I ayant reçu une greffe. De plus, cette analyse suggère que certaines variables semblent associées au niveau de la QV de ces patients, notamment le type de greffe reçu, le retour au travail et la satisfaction du traitement.

Cette étude constitue un exemple des possibilités qu'offre l'analyse de classification hiérarchique. Toutefois, comme la plupart des méthodes quantitatives, l'analyse de classification hiérarchique comporte certaines limites. Le désavantage majeur est qu'il existe une énorme variété de méthodes et d'algorithmes pour effectuer cette analyse. De plus, un même algorithme peut parfois exister sous plusieurs noms. Ainsi, il devient très difficile de $s^{\prime} y$ démêler et de faire les bons choix (Scheibler \& Schneider, 1985). D'ailleurs, cette complexité peut sembler intimidante, particulièrement pour les chercheurs en sciences humaines. Cette limite peut par ailleurs être adressée à la plupart des analyses de classification. À cet effet, Blashfield \& Aldenderfer (1988) ont souligné le besoin pressant de développer une base théorique complète et adéquate pour toutes les méthodes de classification. La plupart des chercheurs qui ont développé les diverses méthodes de classification n'ont pas suffisamment explicité les avantages et inconvénients de chacune d'entre elles (Blashfield \& Aldenderfer, 1988). De plus, peu de littérature est disponible sur l'application concrète des méthodes de classification dans la recherche en sciences humaines. Néanmoins, il semble que les méthodes de classification entrent actuellement dans une phase de consolidation dans le domaine de la psychologie (Blashfield \& Aldenderfer, 1988). Ceci permet d'espérer qu'elles seront de plus en plus intégrées, comprises et utilisées à bon escient dans notre discipline.

\section{Références}

Barbaranelli, C. (2001). Evaluating cluster analysis solutions: An application to the Italian NEO Personality Inventory. European Journal of Personality, 16, S45-S55.

Beaulieu-Prévost, D. (2002). Analyse de classification hiérarchique et typologie des rêveurs. Manuscrit nonpublié.

Blashfield, R.K., \& Aldenderfer, M.S. (1988). The methods and problems of cluster analysis. Dans J.R. Nesselroad (Éd.), Handbook of multivariate experimental psychology, $2^{\text {nd }}$ ed. (447-473). New York : Plenum.

Breckenridge, J.N. (1989). Replicating cluster analysis: Method, consistency, and validity. Multivariate Behavioral Research, 24, 147-161.

Derogatis, L.R. (1986). The psychosocial adjustment to illness scale-self report. Journal of Psychosomatic Research, 30, 77-91.

Gross, C.R., Limwattananon, C., Matthees, B., Zehrer, J.L., \& Savik, K. (2000). Impact of transplantation on quality of life in patients with diabetes and renal dysfunction. Transplantation, 70(12), 1736-1746.

Kos, A.J.\& Psenicka, C. (2000). Measuring similarity across methods. Psychological reports, 86, 858-862.

Knight, R.J., \& Daly, L. (2004). The impact of pancreas transplantation on patient employment opportunities. Clinical transplantation, 18(1), 49-52.

Le diabète et les maladies rénales. Fondation Canadienne $d u$ Rein. Tiré en ligne le 12 juillet 2004, au http://www.rein.ca/francais/publications/brochures/di abete/diabete.htm

Lorr, M. (1983). Cluster analysis for social scientists. San Francisco : Jossey-Bass.

Milligan, G.W. (1981). A review of Monte Carlo tests of cluster analysis. Multivariate Behavioral Research, 16, 379-407.

Morey, L.C., Blashfield, R.K. \& Skinner, H.A. (1983). A comparison of cluster analysis techniques within a sequential validation framework. Multivariate Behavioral Research, 18, 309-329.

Rapkin, B.D. \& Luke, D.A. (1993). Cluster analysis in community research: Epistemology and practice. American Journal of Community Research, 21, 247-277.

Scheibler, D \& Schneider, W. (1985). Monte Carlo tests of the accuracy of cluster analysis algorithms. Multivariate Behavioral Research, 20, 283-304.

Skinner, H.A. (1981). Toward the integration of classification theory and methods. Journal of Abnormal Psychology, 20, 68-87.

Sureshkumar, K.K, Mubin, T., Mikhael, N., Ahmer Kashif, M., Nghiem, D.D., \& Marcus, R.J. (2002). Assessment of quality of life after simultaneous pancreas-kidney transplantation. American Journal of Kidney Diseases, 39(6), 1300-1306. 
Tableau 1. Syntaxe de chacune des étapes à effectuer

\begin{tabular}{ll}
\hline Étape & Syntaxe \\
\hline $\mathbf{1}$ Constituer les profils & CLUSTER paishea paisvoc paisdom paissex paisfam paissoc paispsy \\
& /METHOD WARD \\
/MEASURE= SEUCLID \\
/PRINT SCHEDULE CLUSTER(2) \\
/PLOT VICICLE \\
/SAVE CLUSTER(2). \\
\hline 2 Validation des classes & MANOVA paishea paisvoc paisdom paissex paisfam paissoc paispsy BY clu1 (1, 2).
\end{tabular}

Tableau 2. Distinction des profils de Qualité de Vie (QV) sur les variables internes

\begin{tabular}{|c|c|c|c|c|c|}
\hline \multicolumn{3}{|c|}{ Variables internes } & \multicolumn{2}{|c|}{ Comparaison des profils } & Résultat \\
\hline \multicolumn{3}{|l|}{ Attitude face aux soins de santé } & \multicolumn{2}{|c|}{ Profil $1>$ Profil 2} & $F(1,41)=13.94, p=.001^{*}$ \\
\hline \multicolumn{3}{|c|}{ Adaptation à l'environnement domestique } & \multicolumn{2}{|c|}{ Profil $1>$ Profil 2} & $F(1,41)=34.08, p=.000^{*}$ \\
\hline \multicolumn{3}{|c|}{ Adaptation à l'environnement occupationnel } & \multicolumn{2}{|c|}{ Profil $1>$ Profil 2} & $F(1,41)=85.55, p=.000^{*}$ \\
\hline \multicolumn{3}{|c|}{ Adaptation à l'environnement social } & \multicolumn{2}{|c|}{ Profil $1>$ Profil 2} & $F(1,41)=78.75, p=.000^{*}$ \\
\hline \multicolumn{3}{|l|}{ Satisfaction sexuelle } & \multicolumn{2}{|c|}{ Profil $1>$ Profil 2} & $F(1,41)=33.24, p=.000^{*}$ \\
\hline \multicolumn{3}{|l|}{ Relations avec la famille élargie } & \multicolumn{2}{|c|}{ Profil $1>$ Profil 2} & $F(1,41)=2.31, p=.14$ \\
\hline \multicolumn{3}{|l|}{ Détresse psychologique } & \multicolumn{2}{|c|}{ Profil $1>$ Profil 2} & $F(1,41)=11.58, p=.001^{*}$ \\
\hline \multirow{2}{*}{\multicolumn{6}{|c|}{$\begin{array}{l}\text { Note. Un score plus élevé signifie un niveau d'adaptation plus faible; Profil 1: QV faible; Profil } 2 \\
\text { *: Différence significative en fonction du seuil établi }(\mathrm{p}<.05) \\
\text { Tableau 3. Comparaison des profils de Qualité de Vie }(\mathrm{QV}) \text { sur les variables externes }\end{array}$}} \\
\hline & & & & & \\
\hline \multicolumn{6}{|l|}{ ANOVAs } \\
\hline Variable externe & \multicolumn{2}{|c|}{ Profil } & \multicolumn{2}{|c|}{ M (É.T.) } & tat \\
\hline \multirow[t]{2}{*}{ Complications Rapportées } & \multicolumn{2}{|c|}{1} & \multicolumn{2}{|c|}{$6.0(2.66)$} & $8)=0.14, p=.71$ \\
\hline & \multicolumn{2}{|l|}{2} & \multicolumn{2}{|c|}{$6.36(2.78)$} & \\
\hline \multirow[t]{2}{*}{ Nombre de Réhospitalisations } & \multicolumn{2}{|l|}{1} & \multicolumn{2}{|c|}{$0.92(1.08)$} & 1) $=3.71, p<.06$ \\
\hline & \multicolumn{2}{|l|}{2} & \multicolumn{2}{|c|}{$2.19(2.18)$} & \\
\hline \multirow[t]{2}{*}{ *Satisfaction du traitement } & \multirow{2}{*}{\multicolumn{2}{|c|}{$\begin{array}{l}1 \\
2\end{array}$}} & \multicolumn{2}{|c|}{$6.0(11.42)$} & $8)=6.15, p<.02$ \\
\hline & & & 6.3 & $(8.32)$ & \\
\hline Crosstabs & & il 1 & Pro & $\begin{array}{l}\text { il } 2 \\
\text { élevée } n=31 \text { ) }\end{array}$ & \\
\hline Variables externes & $\mathbf{N}$ & $\%$ & $\mathbf{N}$ & $\%$ & tat \\
\hline Type de greffe & & & & & \\
\hline Rénale & 9 & 75 & 9 & 29 & $N=43)=7.51, p<.01$ \\
\hline Double & 3 & 25 & 22 & 71 & \\
\hline Travaille actuellement & & & & & \\
\hline Oui & 2 & 18.2 & 17 & 54.8 & $N=42)=4.41, p<.05$ \\
\hline Non & 9 & 81.8 & 14 & 45.2 & \\
\hline
\end{tabular}

Note. M (É. T.): Moyenne (Écart type); Profil 1 : QV psychosociale faible ; Profil 2 : QV psychosociale élevée.

*: Un score faible signifie un haut niveau de satisfaction 